\title{
Indirect dark matter searches with neutrinos from the Galactic Centre region with the ANTARES and KM3NeT telescopes
}

\section{Sara Rebecca Gozzini ${ }^{a, *}$ on behalf of the ANTARES and KM3NeT Collaborations}

(a complete list of authors can be found at the end of the proceedings)

\author{
${ }^{a}$ IFIC (CSIC - Universitat de València) \\ Carrer del Catedrátic José Beltrán Martinez, 2, 46980, Paterna, Spain \\ E-mail: srgozzini@km3net.de
}

\begin{abstract}
An anomalous flux of neutrinos produced in hypothetical annihilations or decays of dark matter inside a source would produce a signal observable with neutrino telescopes. As suggested by observations, a conspicuous amount of dark matter is believed to accumulate in the centre of our Galaxy, which is in neat visibility for the Mediterranean underwater telescopes ANTARES and KM3NeT. Searches have been conducted with a maximum likelihood method to identify the presence of a dark matter signature in the neutrino flux measured by ANTARES. Results of all-flavour searches for WIMPs with masses from $50 \mathrm{GeV} / \mathrm{c}^{2}$ up to $100 \mathrm{TeV} / \mathrm{c}^{2}$ over the whole operation period from 2007 to 2020 are presented here. Alternative scenarios which propose a dark matter candidate in the heavy sector extensions of the Standard Model would produce a clear signature in the ANTARES telescope, that can exploit its view of the Galactic Centre up to high energies. The presentation of Galactic Centre searches is completed with ongoing analyses and future potential of the KM3NeT telescope, in phased construction in the Mediterranean Sea.
\end{abstract}

$37^{\text {th }}$ International Cosmic Ray Conference (ICRC 2021)

July 12th-23rd, 2021

Online - Berlin, Germany

\footnotetext{
*Presenter
} 


\section{Physics case}

Astrophysical messengers such as neutrinos and photons can be trackers of the annihilation of dark matter particles inside a celestial object. Dark matter makes up to $27 \%$ of the Universe massenergy budget, fact which is inferred through macroscopic observations: cosmology and gravitation. It is a natural approach to seek a microscopic theory of dark matter, fitting it in an extension of the Standard Model [1]. This new type of matter could be composed by new elementary particles that evade detection due to their weak interaction with baryonic matter. All microscopic searches for a fundamental dark matter particle have until now come up empty-handed. To enhance the possibility of detecting such low interaction rates, one turns to accumulation points in astrophysical ambient where to find the highest density of dark matter, gravitationally bound. Those regions are indicated as dark matter sources, considerably more extended than classical astrophysical emitters. The amount of dark matter inside an accumulation point is characterised using the $J$-factor:

$$
J=\int_{\Omega} d \Omega \int_{l} \rho^{2}(r(\theta, \phi)) d l
$$

The $J$-factor expresses the dark matter density integrated over a viewing angle $\Omega$ and along a line of sight $l$. The shape of the dark matter halo $\rho(r(\theta, \phi))$ is, in turn, described with models built around astrophysical data, or taking input from N-body simulations, such as Navarro-Frenk-White (NFW) [2] which is used through this analysis. The CLUMPY software [3] provides numerical evaluation of the J-Factors to quantify the amount of dark matter in a given field of view around a source.

To proceed in the evaluation of the neutrino yield from a dark matter pair-annihilation process, the context is from here on restricted to cold (non relativistic), weakly interacting massive particles (WIMPs) gravitationally bound into the centre of the Milky Way, that can pair annihilate as

$$
\text { WIMP WIMP } \rightarrow \text { intermediate channel } \rightarrow v \bar{v} .
$$

The energy distribution of annihilations of classical WIMPs between $\sim 1 \mathrm{GeV}$ and $100 \mathrm{TeV}$ is described using PYTHIA, and made ready-to-use in the PPPC4 framework [4], as detailed in section 4.1. There is no prior knowledge on the WIMP mass, that is considered in this analysis from $50 \mathrm{GeV} / c^{2}$ to $100 \mathrm{TeV} / c^{2}$. A neutrino telescope performs a measurement of the outcoming event rate from a scattering as in Equation 2, converted into a limit on the number of scatterings, and assuming that the dark matter content of the source is known, into a limit on the velocity-averaged cross-section for annihilation. Indeed, cold dark matter particles move towards one another with velocity $v \ll c$, hence the cross section is accessible only averaged over the particle velocity distribution.

\section{Instruments}

ANTARES is a Cherenkov neutrino detector located underwater in the Mediterranean Sea 40 $\mathrm{km}$ offshore from Toulon. It is composed of 12 detection lines with a length of 450 metres, anchored to the seabed at a depth of about 2500 metres. Those lines host photomultiplier tubes enclosed in 885 optical modules, which instrument about $0.1 \mathrm{~km}^{2}$ of water. ANTARES is served by the shore station of Les Sablettes and the control room at the Michel Pacha Institute at La Seyne, France. 
For a detailed technical description see [5]. The ANTARES telescope was initially designed for neutrino astronomy; its layout is optimised to detect energies between a few tenths of $\mathrm{GeV} / \mathrm{c}^{2}$ and $10^{8} \mathrm{GeV} / \mathrm{c}^{2}$. This energy window is exploited for dark-matter searches in its lower half, from threshold to $100 \mathrm{TeV} / \mathrm{c}^{2}$. From its geographical position at $42^{\circ} 48^{\prime} \mathrm{N}, 6^{\circ} 10^{\prime} \mathrm{E}$, this instrument has a coverage of Galactic Centre for about $70 \%$ of the time.

The technology know-how and operation expertise that lead to the excellent performance of ANTARES (long-term data taking, very high duty cycle) have flown into conceiving the KM3NeT infrastructure. KM3NeT is a modular network of detectors as of now being deployed in the Mediterranean Sea with a phased installation scheme. The configuration currently in construction hosts one cubic kilometre detector, KM3NeT-ARCA (Astroparticle Research with Cosmics in the Abyss), conceived to catch low astrophysical fluxes, and one dense detector of smaller size, KM3NeTORCA (Oscillation Research with Cosmics in the Abyss), to study the properties of atmospheric neutrinos with unprecedented statistics [6]. Although the main goals of these instruments are the identification of neutrino sources (large volume, excellent angular resolution) and the precision measurement of neutrino mass ordering and oscillation parameters (very high statistics), both detectors are potentially suited to perform dark-matter searches. Geographically, KM3NeT-ARCA is placed offshore the Southern coast of Sicily, and KM3NeT-ORCA close to the ANTARES site: as well as ANTARES, both KM3NeT detectors ensure a good visibility of the Galactic Centre. All the KM3NeT detectors have the same technology and layout. Their geometry foresees 115 lines, with different spacing: $36 \mathrm{~m}$ between optical modules and $90 \mathrm{~m}$ inter-line spacing for KM3NeTARCA (that hosts two such blocks); $9 \mathrm{~m}$ between optical modules and $20 \mathrm{~m}$ inter-line spacing for KM3NeT-ORCA. KM3NeT-ARCA is connected to the shore station of Capo Passero (Italy) while KM3NeT-ORCA uses the same facilities serving the ANTARES supply and DAQ. Currently both detectors consist of 6 lines each, connected and already recording data.

\section{Data}

The data presented in this analysis were recorded with ANTARES between January 2007 and February 2020. One data event appears in ANTARES as a pattern of light hits with timing information, caused by a lepton embedded in its Cherenkov cone crossing the detector. Through reconstruction of arrival direction, energy and topology of the event it is possible to backtrack the variables of the mother neutrino that yield this lepton through a scattering process in the vicinity of the instrumented volume. Such reconstruction is realised in ANTARES through three different algorithms that we name here AAFit (for track-line events illuminating more than one detector line), BBFit (illuminating only one line) and TFit (for shower-like events). In this analysis, both tracks and shower candidates are included. The livetime of this analysis is 3845 days.

Data are preselected upon quality criteria using indicators belonging to each fit, as summarized in Table 1. Here the subscript $A A, B B, T$ stay for multi-line tracks, single-line tracks and shower variables respectively. The reference sample obtained with values as in Table 1 contains 11174 tracks and 225 showers. The number of light hits in each event is directly connected with the energy (proportional, in turn, to the amount of light deposited). ANTARES ensures a reliable energy reconstruction only for mid- to high energy tracks (muon length > 380 metres), reason why the dark matter analyses do not make use of the standard energy estimator. However, using the 


\begin{tabular}{|l|l|}
\hline High track likelihood & $\Lambda_{\mathrm{AA}}>-5.2$ or $\chi_{B B}^{2}<1.4$ \\
Small angular error & $\beta_{\mathrm{AA}}<1^{\circ}$ \\
Upgoing & $\cos \left(\theta_{\mathrm{AA}}\right)>0$ or $\cos \left(\theta_{\mathrm{BB}}\right)>0$ \\
\hline \hline High shower likelihood & $\operatorname{lik}_{T}>50, \mathrm{lik}_{D}>0.3$ \\
Time residual fit matches shower geometry & $M_{T}<1000$ \\
Small enough angular error & $\beta_{T}<30^{\circ}$ \\
Upgoing & $\cos \left(\theta_{T}\right)>0.0$ \\
Vertex confinement & $\sqrt{x_{T}^{2}+y_{T}^{2}}<300 \mathrm{~m},\left|z_{T}\right|<250 \mathrm{~m}$ \\
\hline
\end{tabular}

Table 1: Selection criteria adopted for track candidates (top block) and shower candidates (bottom block).

number of hits $N_{\text {HITS }}$ as an energy proxy, it is possible to implement an additional energy dependent selection. This wants to best exploit the shape of the WIMP annihilation spectra, in particular for such channels as WIMP WIMP $\rightarrow v \bar{v}$ where a large fraction of events piles up at the right end of the spectrum (this feature can be seen in Figure 1). A cut in $N_{\text {HITS }}$ is varied choosing between values corresponding to a reduction of the total acceptance of $90 \%, 75 \%, 50 \%$ and $25 \%$ respectively. The values leading to best sensitivity are used. A set of simulated data has been produced in ANTARES in correspondence with the environmental and trigger conditions of each data run in the DAQ. This Monte Carlo simulation is used in this search to reproduce the dark matter signal through appropriate weights, as detailed in section 4.1.

\section{Analysis procedure}

This search is structured as a hypothesis test. A signal hypothesis $H 1$, where a cluster of $n_{s}$ neutrino events around the Galactic Centre region come from pair annihilation of dark matter, is to be discriminated from the null hypothesis $H_{0}$ where all $n$ events in the sample are neutrinos originated in the Earth's atmosphere. The number of expected signal events $n_{s}$ is a priori unknown and is varied between 1 and 50, identifying the most plausible value with an unbinned maximum likelihood method. To conduct the hypothesis test, a test statistic $T S$ is defined as likelihood ratio. Sensitivity is obtained as the average upper limit from pseudo-experiments, which allows to optimise cuts for best sensitivity. Until this point, the data set is blind (i.e. right-ascension shuffled). Upon unblinding, upper limits at $90 \%$ C.L. are set.

\subsection{WIMP pair annihilation signal at ANTARES}

The specific signal that would appear in ANTARES is obtained reweighting Monte Carlo simulated events to match the energy distribution a WIMP pair collision, and distributing them around the position of the Galactic Centre according to the shape of a dark matter halo profile, folding in the detector resolution. Input used in this analysis are energy distributions from the PPPC4 tables [4] for the single WIMP collision, and the NFW halo profile [2]. Probability density functions (PDFs) for the signal are obtained and input in the likelihood. In this analysis, five channels are considered with independent $100 \%$ branching ratio each:

$$
\mathrm{WIMP} \text { WIMP } \rightarrow \tau^{+} \tau^{-} \rightarrow v \bar{v}, \mu^{+} \mu^{-} \rightarrow v \bar{v}, b \bar{b} \rightarrow v \bar{v}, W^{+} W^{-} \rightarrow v \bar{v}, v \bar{v}
$$



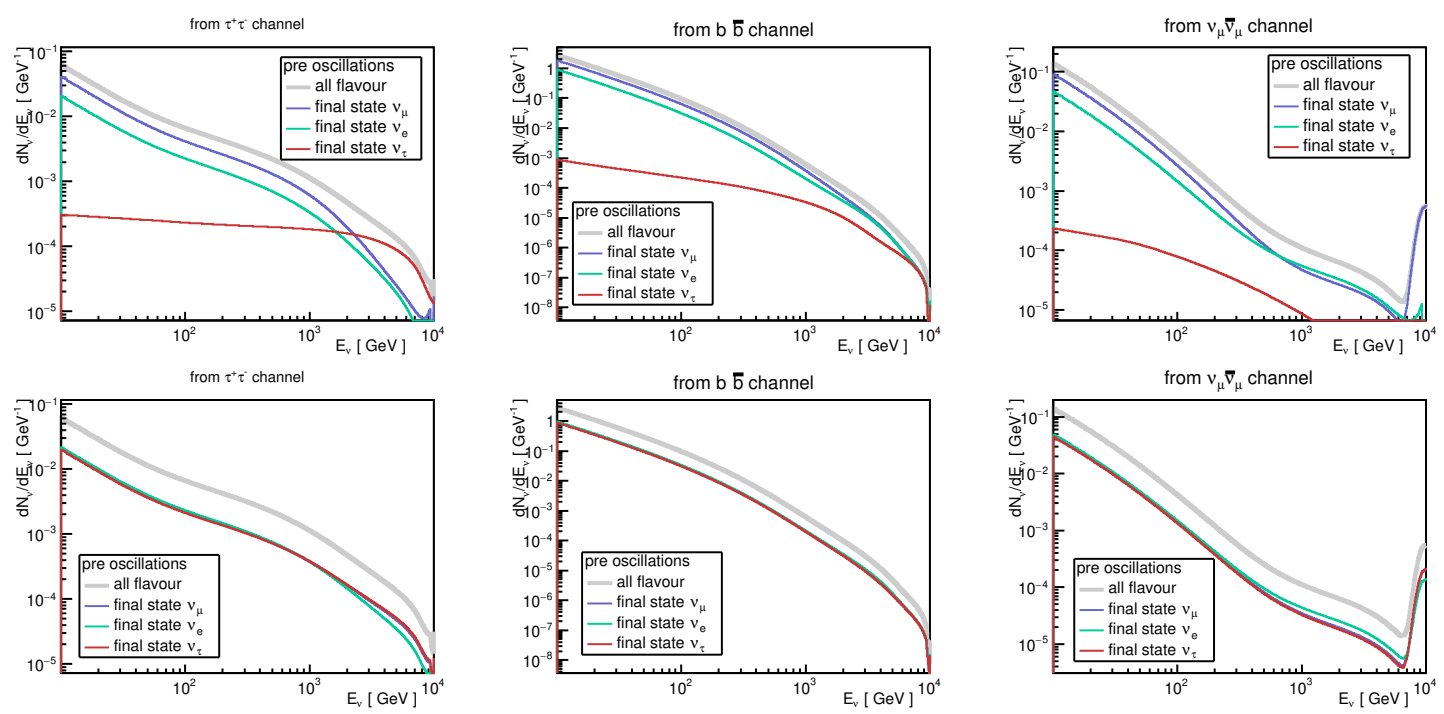

Figure 1: Energy distributions of a $10 \mathrm{TeV}$ WIMP pair-annihilating into three benchmark channels $\tau^{+} \tau^{-}, b \bar{b}, v \bar{v}$, as obtained with PPPC4 [4]. The upper panels display the spectra at the Galactic Centre, the bottom panel at detection point after flavour oscillations in the long baseline approximation.

The corresponding energy distributions are displayed in the upper panel of Figure 1. The complete process takes place inside the source, which the final products leave already as neutrinos, allowing to neglect energy distortion due to absorption. The neutrino yield obtained with PPPC4 is then oscillated in the long baseline approximation between the source and the detection point to obtain the distributions in the bottom panel of Figure 1.

\subsection{Background}

Once eliminated the events with low reconstruction quality, the spurious coincidences (electronic noise, bioluminescence) and the atmospheric muons (through the Earth screen cut), the background left consists of atmospheric neutrinos and wrongly reconstructed atmospheric muons. This is an irreducible background that distributes isotropically in right ascension, and follow a decreasing power-law energy spectrum. In this analysis, background is not simulated with Monte Carlo but directly obtained from data after random shuffling the right ascension coordinate. This ensures to wash out any possible signal clusters present in the data.

\subsection{Hypothesis Test}

The best sensitivity in reach with this search is computed using pseudo-experiments (simulated skymaps) to identify the final cut combination. A likelihood function is constructed per each simulated skymap using the signal and background $\operatorname{PDFs} \mathcal{S}, \mathcal{B}$, the number of injected signal $n_{s}$, and the number of background events $n_{b g}$. The total amount of events $n=n_{s}+N_{b g}$ corresponds to the number of real data events:

$$
\log \mathcal{L}\left(n_{s}\right)=\sum_{i=1}^{N} \log \left[n_{s} \mathcal{S}\left(\psi_{i}, N_{\mathrm{HITS}}^{i}, q_{i}\right)+n_{b g} \mathcal{B}\left(\delta_{i}, N_{\mathrm{HITS}}^{i}, q_{i}\right)\right]-n_{b g}-n_{s}
$$


In this analysis, both track and shower candidates are included. The number of signal and background events is split into a relative fraction of tracks and showers that reflect the acceptance ratio, per each WIMP mass and annihilation channel considered. Likelihood maximisation returns the most likely number of events $n_{s}^{*}$ found to belong to a cluster around the fixed coordinates of the Galactic Centre $(\alpha, \delta)=\left(266^{\circ},-29^{\circ}\right)$. This is repeated for $10^{4}$ pseudo-experiments for each parameter choice. The significance of a signal cluster is established by the test statistics TS, function of the ratio between maximum likelihood and pure background likelihood

$$
T S=-\log \frac{\mathcal{L}\left(n_{s}^{*}\right)}{\mathcal{L}\left(n_{s}=0\right)} .
$$

The number of events in each set of pseudo-experiments is subject to fluctuations following a Poisson distribution. To include this effect, a transformation through a Poisson function $\mathcal{P}$ is performed, returning the TS as a function of the Poissonian mean $\mu$ as $P(T S(\mu))=P\left(T S\left(n_{s}^{*}\right)\right) \times \mathcal{P}\left(n_{s}, \mu\right)$. Finally, a 15\% systematics on the number of detected events is expected with ANTARES [7]. This effect is included folding the above equation with a Gaussian smearing of $15 \%$ width. Following Neyman's prescription [8], an average upper limit on the number of signal events is firstly computed from the background median test statistics $\overline{T S}_{0}$, compared with each distribution $P(T S)$ for each pseudo-experiment set. The sensitivity is defined as the $90 \%$ C.L. upper limit for a measurement coinciding with the median of the background TS distribution.

\subsection{Analysis acceptance}

The effective area represents the equivalent surface of the detector once accounted for efficiency losses due to geometry, trigger efficiency and the analysis cuts. As both the effective area and the WIMP annihilation spectra are energy dependent, folding those two distributions gives a measurement of the acceptance, or capability of the detector to take an event. The acceptance is defined as the effective area modulated through each annihilation mode spectrum $d N_{v} / d E_{v}$, integrated between the energy threshold of the detector and the WIMP mass $M$ :

$$
\mathcal{A}(M)=\int_{0}^{M} A_{e f f}^{v}\left(E_{v}\right) \frac{d N_{\nu}\left(E_{v}\right)}{d E_{v}} d E_{v}+A_{e f f}^{\bar{v}}\left(E_{\bar{v}}\right) \frac{d N_{\bar{\nu}}\left(E_{\bar{\nu}}\right)}{d E_{\bar{v}}} d E_{\bar{v}}
$$

Through acceptance and livetime, a measurement (upper limit) of number of events is converted into flux of particles reaching the detector by $\Phi_{\nu+\bar{\nu}}=\frac{\mu_{90}}{\mathcal{A} t}$.

\section{Results and discussion}

A cut mask corresponding to the best sensitivity values is applied to the data, once retrieved the original right ascension coordinate. A combination of best cuts is obtained independently per WIMP mass and annihilation channel. To unblind, each data event is flagged as a track or shower according to the quality variables described in Table 1 , and the likelihood is evaluated with the corresponding PDFs. Upon unblinding, the TS observed in the 2007-2020 all-flavour data set is compatible with the background hypothesis for all combinations of WIMP masses and annihilation channels considered. The upper limit on the flux of outcoming events from WIMP WIMP scattering 


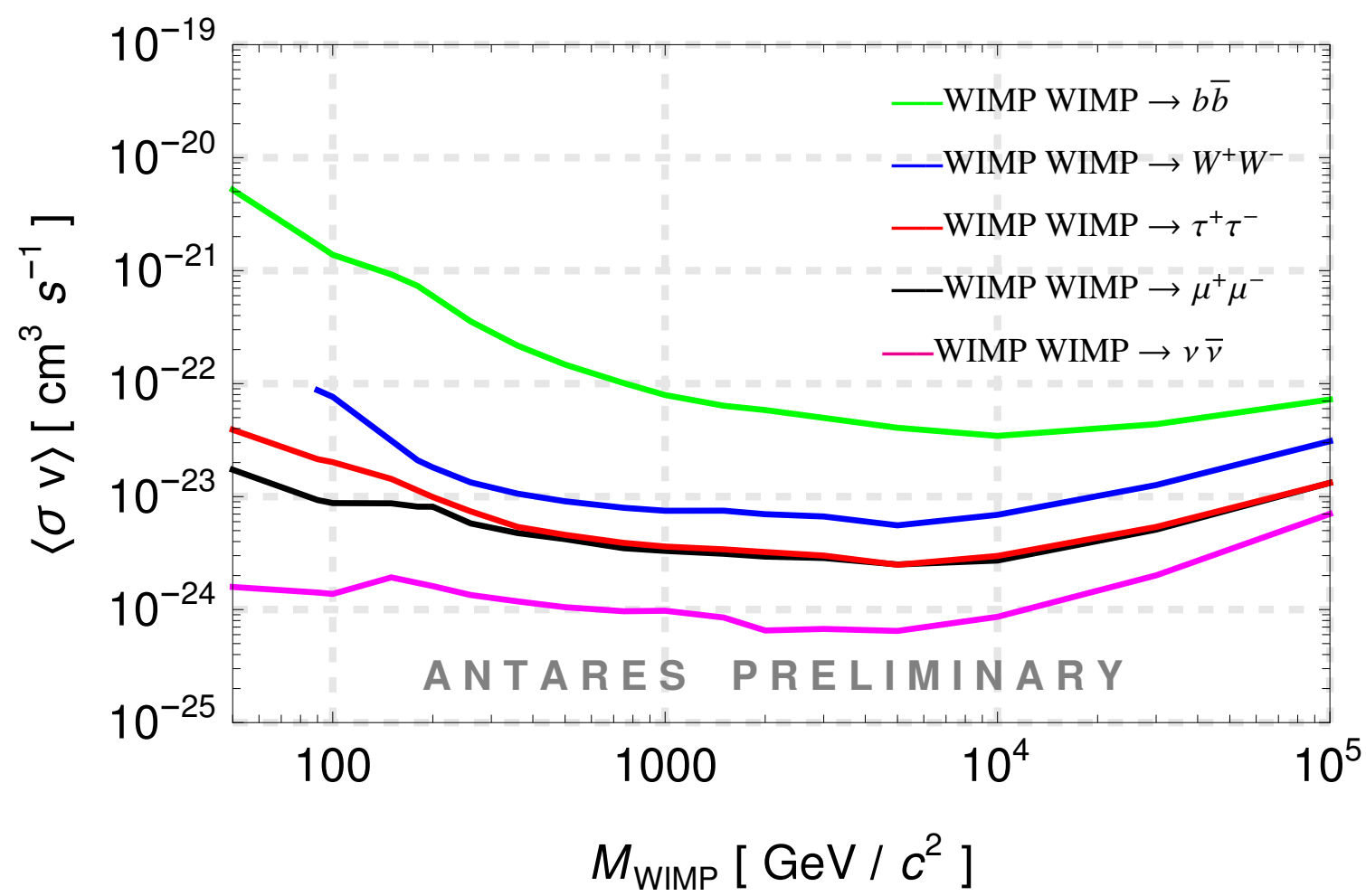

Figure 2: Upper limits at 90\% C.L. on the thermally averaged cross-section for WIMP pair annihilation as a function of the WIMP candidate mass set with 14 years of ANTARES data, shown for five independent annihilation channels (each with $100 \%$ branching ratio) and NFW halo model [2].

is converted into a limit o the velocity-averaged cross section with

$$
\frac{d \Phi\left(E_{v}\right)}{d E_{v}}=\frac{1}{4 \pi M^{2}} \frac{\langle\sigma v\rangle}{2} \frac{d N\left(E_{v}\right)}{d E_{v}} J .
$$

Upper limits at $90 \%$ C.L. based on this non-observation of signal from dark matter annihilations are shown in Figure 2. The best limits are obtained with the direct annihilation into two neutrinos, in which this channel displays the majority of events in the high-energy tail of the WIMP annihilation spectrum. Figure 3 shows the sensitivity that would be reached with KM3NeT-ARCA after 1 year, which is particularly promising for WIMP masses above $10^{4} \mathrm{GeV} / \mathrm{c}^{2}$ when placed in context with the same measurement $\left(\tau^{+} \tau^{-}\right.$channel) performed with other experiments.

\section{References}

[1] G. Bertone. Particle Dark Matter : Observations, Models and Searches (2010).

[2] J. F. Navarro, C. S. Frenk and S. D. M. White, Astrophys. J. 462 (1996), 563-575

[3] M. Hütten, C. Combet, D. Maurin et al, https://clumpy.gitlab.io/CLUMPY

[4] M. Cirelli, G. Corcella, A. Hektor, G. Hutsi, M. Kadastik, P. Panci, M. Raidal, F. Sala and A. Strumia, JCAP 03 (2011), 051 [erratum: JCAP 10 (2012), E01] 


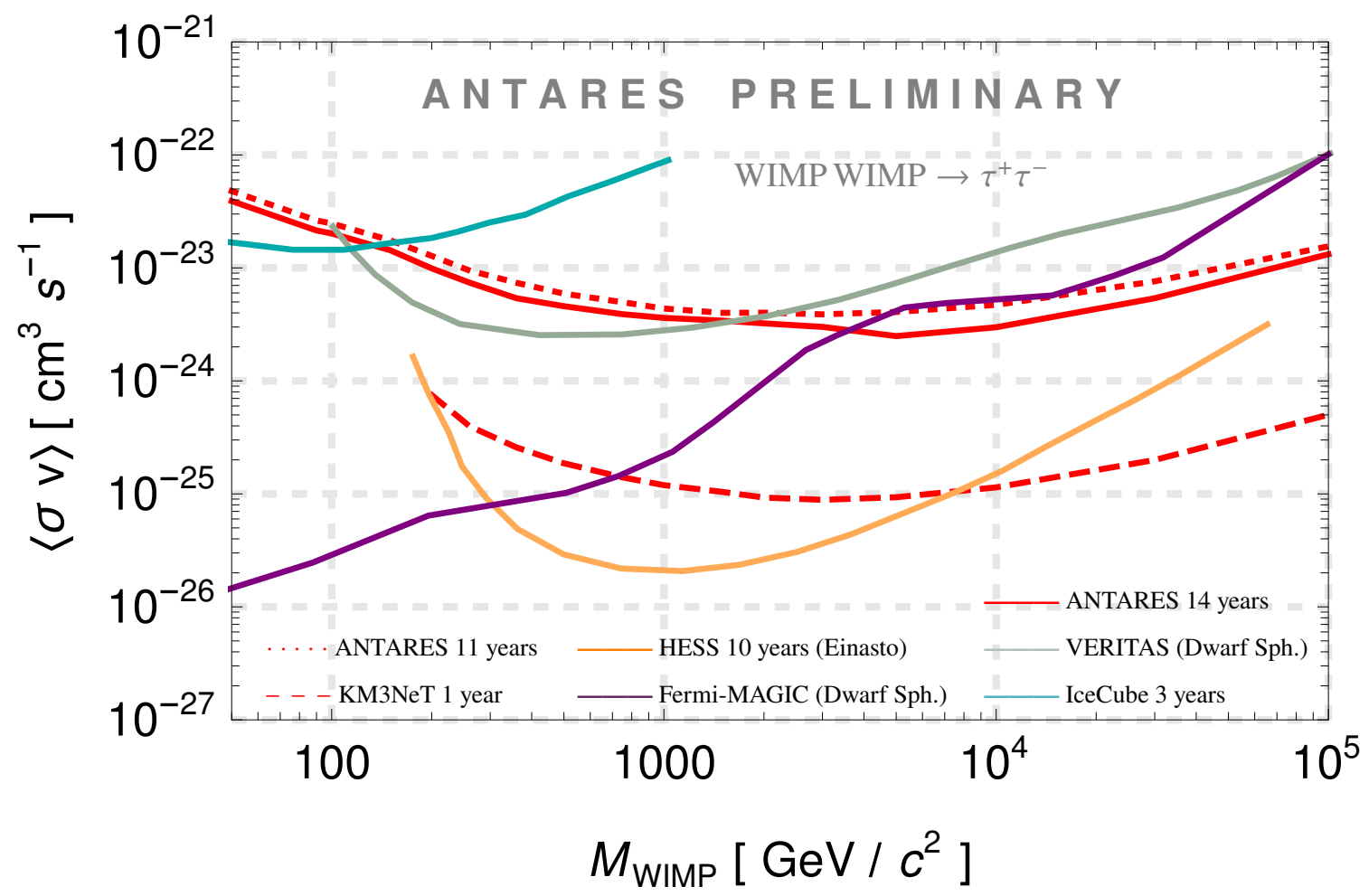

Figure 3: Upper limits at $90 \%$ C.L. on the thermally averaged cross-section for WIMP pair annihilation as a function of the WIMP candidate mass set with 14 years of ANTARES data, in comparison with the same measurement from IceCube [9], HESS [10], VERITAS [11] and Fermi-LAT+MAGIC [12], for the $\tau^{+} \tau^{-}$ channel and NFW dark matter halo [2], unless specified. The sensitivity that could be reached with KM3NeT in 1 year is shown in dashed red.

[5] M. Ageron et al. [ANTARES], Nucl. Instrum. Meth. A 656 (2011), 11-38

[6] S. Adrian-Martinez et al. [KM3Net], J. Phys. G 43 (2016) no.8, 084001

[7] A. Albert et al. [ANTARES], Phys. Rev. D 96 (2017) no.8, 082001

[8] J. Neyman, Lectures and Conferences on Mathematical Statistics (1938).

[9] M. G. Aartsen et al. [IceCube], Eur. Phys. J. C 77 (2017) no.9, 627

[10] H. Abdallah et al. [H.E.S.S.], Phys. Rev. Lett. 117 (2016) no.11, 111301

[11] S. Archambault et al. [VERITAS], Phys. Rev. D 95 (2017) no.8, 082001

[12] M. L. Ahnen et al. [MAGIC and Fermi-LAT], JCAP 02 (2016), 039 


\section{Full Authors List: ANTARES Collaboration}

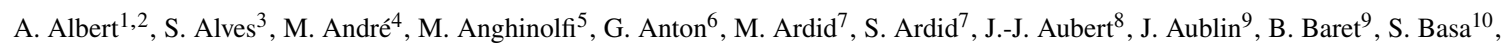
B. Belhorma ${ }^{11}$, M. Bendahman ${ }^{9,12}$, V. Bertin ${ }^{8}$, S. Biagi ${ }^{13}$, M. Bissinger $^{6}$, J. Boumaaza ${ }^{12}$, M. Bouta ${ }^{14}$, M.C. Bouwhuis ${ }^{15}$, H. Brânzaş ${ }^{16}$, R. Bruijn ${ }^{15,17}$, J. Brunner ${ }^{8}$, J. Busto ${ }^{8}$, B. Caiff ${ }^{5}$, A. Capone ${ }^{18,19}$, L. Caramete ${ }^{16}$, J. Carr ${ }^{8}$, V. Carretero ${ }^{3}$, S. Celli ${ }^{18,19}$, M. Chabab ${ }^{20}$, T. N. Chau ${ }^{9}$, R. Cherkaoui El Moursli ${ }^{12}$, T. Chiarusi ${ }^{21}$, M. Circella $^{22}$, A. Coleiro ${ }^{9}$, M. Colomer-Molla ${ }^{9,3}$, R. Coniglione ${ }^{13}$, P. Coyle $^{8}$, A. Creusot $^{9}$, A. F. Díaz ${ }^{23}$, G. de Wasseige ${ }^{9}$, A. Deschamps ${ }^{24}$, C. Distefano ${ }^{13}$, I. Di Palma ${ }^{18,19}$, A. Domi ${ }^{15,17}$, C. Donzaud ${ }^{9,25}$, D. Dornic ${ }^{8}$, D. Drouhin ${ }^{1,2}$, T. Eberl ${ }^{6}$, T. van Eeden ${ }^{15}$, D. van Eijk ${ }^{15}$, N. El Khayati ${ }^{12}$, A. Enzenhöfer ${ }^{8}$, P. Fermani ${ }^{18,19}$, G. Ferrara ${ }^{13}$, F. Filippini ${ }^{21,26}$, L.A. Fusco ${ }^{8}$, Y. Gatelet ${ }^{9}$, P. Gay ${ }^{27,9}$, H. Glotin ${ }^{28}$, R. Gozzini ${ }^{3}$, R. Gracia Ruiz ${ }^{15}$, K. Graf ${ }^{6}$, C. Guidi ${ }^{5,29}$, S. Hallmann 6 , H. van Haren ${ }^{30}$, A.J. Heijboer ${ }^{15}$, Y. Hello ${ }^{24}$, J.J. Hernández-Rey ${ }^{3}$, J. Höß1 ${ }^{6}$, J. Hofestädt ${ }^{6}$, F. Huang ${ }^{8}$, G. Illuminati ${ }^{9}, 21,26$, C.W James $^{31}$, B. Jisse-Jung ${ }^{15}$, M. de Jong ${ }^{15,32}$, P. de Jong ${ }^{15}$, M. Kadler ${ }^{33}$, O. Kalekin ${ }^{6}$, U. Katz ${ }^{6}$, N.R. Khan-Chowdhury ${ }^{3}$, A. Kouchner ${ }^{9}$, I. Kreykenbohm ${ }^{34}$, V. Kulikovskiy ${ }^{5,36}$, R. Lahmann ${ }^{6}$, R. Le Breton ${ }^{9}$, D. Lefèvre ${ }^{35}$, E. Leonora ${ }^{36}$, G. Levi ${ }^{21,26}$, M. Lincetto ${ }^{8}$, D. Lopez-Coto ${ }^{37}$, S. Loucatos ${ }^{38,9}$, L. Maderer ${ }^{9}$, J. Manczak ${ }^{3}$, M. Marcelin ${ }^{10}$, A. Margiotta ${ }^{21,26}$, A. Marinelli ${ }^{39}$, J.A. Martínez-Mora ${ }^{7}$, K. Melis ${ }^{15,17}$, P. Migliozzi ${ }^{39}$, A. Moussa ${ }^{14}$, R. Muller ${ }^{15}$, L. Nauta ${ }^{15}$, S. Navas ${ }^{37}$, E. Nezri ${ }^{10}$, B. O' Fearraigh $^{15}$, A. Păun ${ }^{16}$, G.E. Păvălaş ${ }^{16}$, C. Pellegrino ${ }^{21,40,41}$, M. Perrin-Terrin ${ }^{8}$, V. Pestel ${ }^{15}$, P. Piattelli ${ }^{13}$, C. Pieterse ${ }^{3}$, C. Poirè ${ }^{7}$, V. Popa ${ }^{16}$, T. Pradier ${ }^{1}$, N. Randazzo ${ }^{36}$, S. Reck $^{6}$, G. Riccobene ${ }^{13}$, A. Romanov ${ }^{5,29}$, A. Sánchez-Losa ${ }^{3,22}$, F. Salesa Greus ${ }^{3}$, D. F. E. Samtleben ${ }^{15,32}$, M. Sanguineti ${ }^{5,29}$, P. Sapienza ${ }^{13}$, J. Schnabel ${ }^{6}$, J. Schumann ${ }^{6}$, F. Schüssler ${ }^{38}$, M. Spurio ${ }^{21,26}$, Th. Stolarczyk ${ }^{38}$, M. Taiuti ${ }^{5,29}$, Y. Tayalati ${ }^{12}$, S.J. Tingay ${ }^{31}$, B. Vallage ${ }^{38,9}$, V. Van Elewyck ${ }^{9,41}$, F. Versari ${ }^{21,26,9}$, S. Viola ${ }^{13}$, D. Vivolo ${ }^{39,43}$, J. Wilms ${ }^{34}$, S. Zavatarelli ${ }^{5}$, A. Zegarelli ${ }^{18,19}$, J.D. Zornoza ${ }^{3}$, and J. Zúñiga ${ }^{3}$

${ }^{1}$ Université de Strasbourg, CNRS, IPHC UMR 7178, F-67000 Strasbourg, France. ${ }^{2}$ Université de Haute Alsace, F-68100 Mulhouse, France. ${ }^{3}$ IFIC - Instituto de Física Corpuscular (CSIC - Universitat de València) c/ Catedrático José Beltrán, 2 E-46980 Paterna, Valencia, Spain. ${ }^{4}$ Technical University of Catalonia, Laboratory of Applied Bioacoustics, Rambla Exposició, 08800 Vilanova i la Geltrú, Barcelona, Spain. ${ }^{5}$ INFN - Sezione di Genova, Via Dodecaneso 33, 16146 Genova, Italy. ${ }^{6}$ Friedrich-Alexander-Universität Erlangen-Nürnberg, Erlangen Centre for Astroparticle Physics, Erwin-Rommel-Str. 1, 91058 Erlangen, Germany. ${ }^{7}$ Institut d'Investigació per a la Gestió Integrada de les Zones Costaneres (IGIC) - Universitat Politècnica de València. C/ Paranimf 1, 46730 Gandia, Spain. ${ }^{8}$ Aix Marseille Univ, CNRS/IN2P3, CPPM, Marseille, France. ${ }^{9}$ Université de Paris, CNRS, Astroparticule et Cosmologie, F-75013 Paris, France. ${ }^{10}$ Aix Marseille Univ, CNRS, CNES, LAM, Marseille, France. ${ }^{11}$ National Center for Energy Sciences and Nuclear Techniques, B.P.1382, R. P.10001 Rabat, Morocco. ${ }^{12}$ University Mohammed V in Rabat, Faculty of Sciences, 4 av. Ibn Battouta, B.P. 1014, R.P. 10000 Rabat, Morocco. ${ }^{13}$ INFN - Laboratori Nazionali del Sud (LNS), Via S. Sofia 62, 95123 Catania, Italy. ${ }^{14}$ University Mohammed I, Laboratory of Physics of Matter and Radiations, B.P.717, Oujda 6000, Morocco. ${ }^{15}$ Nikhef, Science Park, Amsterdam, The Netherlands. ${ }^{16}$ Institute of Space Science, RO-077125 Bucharest, Măgurele, Romania. ${ }^{17}$ Universiteit van Amsterdam, Instituut voor Hoge-Energie Fysica, Science Park 105, 1098 XG Amsterdam, The Netherlands. ${ }^{18}$ INFN - Sezione di Roma, P.le Aldo Moro 2, 00185 Roma, Italy. ${ }^{19}$ Dipartimento di Fisica dell’Università La Sapienza, P.le Aldo Moro 2, 00185 Roma, Italy. ${ }^{20}$ LPHEA, Faculty of Science - Semlali, Cadi Ayyad University, P.O.B. 2390, Marrakech, Morocco. ${ }^{21}$ INFN - Sezione di Bologna, Viale Berti-Pichat 6/2, 40127 Bologna, Italy. ${ }^{22}$ INFN - Sezione di Bari, Via E. Orabona 4, 70126 Bari, Italy. ${ }^{23}$ Department of Computer Architecture and Technology/CITIC, University of Granada, 18071 Granada, Spain. ${ }^{24}$ Géoazur, UCA, CNRS, IRD, Observatoire de la Côte d'Azur, Sophia Antipolis, France. ${ }^{25}$ Université Paris-Sud, 91405 Orsay Cedex, France. ${ }^{26}$ Dipartimento di Fisica e Astronomia dell'Università, Viale Berti Pichat 6/2, 40127 Bologna, Italy. ${ }^{27}$ Laboratoire de Physique Corpusculaire, Clermont Université, Université Blaise Pascal, CNRS/IN2P3, BP 10448, F-63000 Clermont-Ferrand, France. ${ }^{28}$ LIS, UMR Université de Toulon, Aix Marseille Université, CNRS, 83041 Toulon, France. ${ }^{29}$ Dipartimento di Fisica dell'Università, Via Dodecaneso 33, 16146 Genova, Italy. ${ }^{30}$ Royal Netherlands Institute for Sea Research (NIOZ), Landsdiep 4, 1797 SZ 't Horntje (Texel), the Netherlands. ${ }^{31}$ International Centre for Radio Astronomy Research - Curtin University, Bentley, WA 6102, Australia. ${ }^{32}$ Huygens-Kamerlingh Onnes Laboratorium, Universiteit Leiden, The Netherlands. ${ }^{33}$ Institut für Theoretische Physik und Astrophysik, Universität Würzburg, Emil-Fischer Str. 31, 97074 Würzburg, Germany. ${ }^{34}$ Dr. Remeis-Sternwarte and ECAP, Friedrich-Alexander-Universität Erlangen-Nürnberg, Sternwartstr. 7, 96049 Bamberg, Germany. ${ }^{35}$ Mediterranean Institute of Oceanography (MIO), Aix-Marseille University, 13288, Marseille, Cedex 9, France; Université du Sud Toulon-Var, CNRS-INSU/IRD UM 110, 83957, La Garde Cedex, France. ${ }^{36}$ INFN - Sezione di Catania, Via S. Sofia 64, 95123 Catania, Italy. ${ }^{37}$ Dpto. de Física Teórica y del Cosmos \& C.A.F.P.E., University of Granada, 18071 Granada, Spain. ${ }^{38}$ IRFU, CEA, Université Paris-Saclay, F-91191 Gif-sur-Yvette, France. ${ }^{39}$ INFN - Sezione di Napoli, Via Cintia 80126 Napoli, Italy. ${ }^{40}$ Museo Storico della Fisica e Centro Studi e Ricerche Enrico Fermi, Piazza del Viminale 1, 00184, Roma. ${ }^{41}$ INFN - CNAF, Viale C. Berti Pichat 6/2, 40127, Bologna. ${ }^{42}$ Institut Universitaire de France, 75005 Paris, France. ${ }^{43}$ Dipartimento di Fisica dell’Università Federico II di Napoli, Via Cintia 80126, Napoli, Italy. 


\section{Full Author List: KM3NeT Collaboration}

M. Ageron ${ }^{1}$, S. Aiello ${ }^{2}$, A. Albert ${ }^{3,55}$, M. Alshamsi ${ }^{4}$, S. Alves Garre ${ }^{5}$, Z. Aly ${ }^{1}$, A. Ambrosone ${ }^{6,7}$, F. Ameli ${ }^{8}$, M. Andre ${ }^{9}$, G. Androulakis ${ }^{10}$, M. Anghinolfi ${ }^{11}$, M. Anguita ${ }^{12}$, G. Anton ${ }^{13}$, M. $\operatorname{Ardid}^{14}$, S. Ardid $^{14}$, W. Assal ${ }^{1}$, J. Aublin ${ }^{4}$, C. Bagatelas ${ }^{10}$, B. Baret ${ }^{4}$, S. Basegmez du Pree ${ }^{15}$, M. Bendahman ${ }^{4,16}$, F. Benfenati ${ }^{17,18}$, E. Berbee ${ }^{15}$, A. M. van den Berg ${ }^{19}$, V. Bertin ${ }^{1}$, S. Beurthey ${ }^{1}$, V. van Beveren ${ }^{15}$, S. Biagi ${ }^{20}$, M. Billault ${ }^{1}$, M. Bissinger ${ }^{13}$, M. Boettcher ${ }^{21}$, M. Bou Cabo ${ }^{22}$, J. Boumaaza ${ }^{16}$, M. Bouta ${ }^{23}$, C. Boutonnet ${ }^{4}$, G. Bouvet ${ }^{24}$, M. Bouwhuis ${ }^{15}$, C. Bozza ${ }^{25}$, H.Brânzaş ${ }^{26}$, R. Bruijn ${ }^{15,27}$, J. Brunner ${ }^{1}$, R. Bruno ${ }^{2}$, E. Buis ${ }^{28}$, R. Buompane ${ }^{6,29}$, J. Busto ${ }^{1}$, B. Caiffí ${ }^{11}$, L. Caillat ${ }^{1}$, D. Calvo ${ }^{5}$, S. Campion ${ }^{30,8}$, A. Capone ${ }^{30,8}$, H. Carduner $^{24}$, V. Carretero ${ }^{5}$, P. Castaldi ${ }^{17,31}$, S. Celli ${ }^{30,8}$, R. Cereseto ${ }^{11}$, M. Chabab ${ }^{32}$, C. Champion ${ }^{4}$, N. $\mathrm{Chau}^{4}$, A. $\mathrm{Chen}^{33}$, S. Cherubini ${ }^{20,34}$, V. Chiarella ${ }^{35}$, T. Chiarusi ${ }^{17}$, M. Circella ${ }^{36}$, R. Cocimano ${ }^{20}$, J. A. B. Coelho ${ }^{4}$, A. Coleiro ${ }^{4}$, M. Colomer Molla ${ }^{4,5}$, S. Colonges ${ }^{4}$, R. Coniglione ${ }^{20}$, A. Cosquer ${ }^{1}$, P. Coyle ${ }^{1}$, M. Cresta ${ }^{11}$, A. Creusot ${ }^{4}$, A. $\mathrm{Cruz}^{37}$, G. Cuttone ${ }^{20}$, A. D’Amico ${ }^{15}$, R. Dallier ${ }^{24}$, B. De Martino ${ }^{1}$, M. De Palma ${ }^{36,38}$, I. Di Palma ${ }^{30,8}$, A. F. Díaz ${ }^{12}$, D. DiegoTortosa $^{14}$, C. Distefano ${ }^{20}$, A. Domi ${ }^{15,27}$, C. Donzaud ${ }^{4}$, D. Dornic ${ }^{1}$, M. Dörr ${ }^{39}$, D. Drouhin ${ }^{3,55}$, T. Eberl ${ }^{13}$, A. Eddyamoui ${ }^{16}$, T. van Eeden ${ }^{15}$, D. van Eijk ${ }^{15}$, I. El Bojaddaini ${ }^{23}$, H. Eljarrari ${ }^{16}$, D. Elsaesser ${ }^{39}$, A. Enzenhöfer ${ }^{1}$, V. Espinosa ${ }^{14}$, P. Fermani ${ }^{30,8}$, G. Ferrara ${ }^{20,34}$, M. D. Filipović ${ }^{40}$, F. Filippini ${ }^{17,18}$, J. Fransen ${ }^{15}$, L. A. Fusco ${ }^{1}$, D. Gajanana ${ }^{15}$, T. Gal ${ }^{13}$, J. García Méndez ${ }^{14}$, A. Garcia Soto $^{5}$, E. Garçon ${ }^{1}$, F. Garufi ${ }^{6,7}$, C. Gatius ${ }^{15}$, N. Geißelbrecht ${ }^{13}$, L. Gialanella ${ }^{6,29}$, E. Giorgio ${ }^{20}$, S. R. Gozzini ${ }^{5}$, R. Gracia ${ }^{15}$, K. Graf ${ }^{13}$, G. Grella ${ }^{41}$, D. Guderian ${ }^{56}$, C. Guidi ${ }^{11,42}$, B. Guillon ${ }^{43}$, M. Gutiérrez ${ }^{44}$, J. Haefner ${ }^{13}$, S. Hallmann ${ }^{13}$, H. Hamdaoui ${ }^{16}$, H. van Haren ${ }^{45}$, A. Heijboer ${ }^{15}$, A. Hekalo ${ }^{39}$, L. Hennig ${ }^{13}$, S. Henry ${ }^{1}$, J. J. Hernández-Rey ${ }^{5}$, J. Hofestädt ${ }^{13}$, F. Huang ${ }^{1}$, W. Idrissi Ibnsalih ${ }^{6,29}$, A. Ilioni ${ }^{4}$, G. Illuminati ${ }^{17,18,4}$, C. W. James ${ }^{37}$, D. Janezashvili ${ }^{46}$, P. Jansweijer ${ }^{15}$, M. de Jong ${ }^{15,47}$, P. de Jong ${ }^{15,27}$, B. J. Jung ${ }^{15}$, M. Kadler ${ }^{39}$, P. Kalaczyński ${ }^{48}$, O. Kalekin ${ }^{13}$, U. F. Katz ${ }^{13}$, F. Kayzel $^{15}$, P. Keller ${ }^{1}$, N. R. Khan Chowdhury ${ }^{5}$, G. Kistauri ${ }^{46}$, F. van der Knaap ${ }^{28}$, P. Kooijman ${ }^{27,57}$, A. Kouchner ${ }^{4,49}$, M. Kreter ${ }^{21}$, V. Kulikovskiy ${ }^{11}$, M. Labalme ${ }^{43}$, P. Lagier ${ }^{1}$, R. Lahmann ${ }^{13}$, P. Lamare ${ }^{1}$, M. Lamoureux $^{14}$, G. Larosa ${ }^{20}$, C. Lastoria ${ }^{1}$, J. Laurence ${ }^{1}$, A. Lazo ${ }^{5}$, R. Le Breton ${ }^{4}$, E. Le Guirriec ${ }^{1}$, S. Le Stum ${ }^{1}$, G. Lehaut ${ }^{43}$, O. Leonardi ${ }^{20}$, F. Leone ${ }^{20,34}$, E. Leonora ${ }^{2}$, C. Lerouvillois ${ }^{1}$, J. Lesrel ${ }^{4}$, N. Lessing ${ }^{13}$, G. Levi ${ }^{17,18}$, M. Lincetto ${ }^{1}$, M. Lindsey Clark ${ }^{4}$, T. Lipreau ${ }^{24}$, C. LLorens Alvarez ${ }^{14}$, A. Lonardo ${ }^{8}$, F. Longhitano ${ }^{2}$, D. Lopez-Coto ${ }^{44}$, N. Lumb ${ }^{1}$, L. Maderer ${ }^{4}$, J. Majumdar ${ }^{15}$, J. Mańczak ${ }^{5}$, A. Margiotta ${ }^{17,18}$, A. Marinelli ${ }^{6}$, A. Marini ${ }^{1}$, C. Markou $^{10}$, L. Martin ${ }^{24}$, J. A. Martínez-Mora ${ }^{14}$, A. Martini ${ }^{35}$, F. Marzaioli ${ }^{6,29}$,

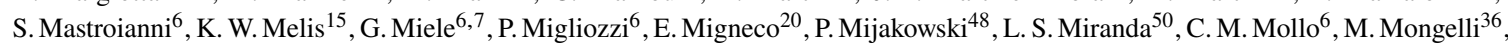
A. Moussa ${ }^{23}$, R. Muller ${ }^{15}$, P. Musico ${ }^{11}$, M. Musumeci ${ }^{20}$, L. Nauta ${ }^{15}$, S. Navas ${ }^{44}$, C. A. Nicolau ${ }^{8}$, B. Nkosi ${ }^{33}$, B. Ó Fearraigh ${ }^{15,27}$, M. O'Sullivan ${ }^{37}$, A. Orlando ${ }^{20}$, G. Ottonello ${ }^{11}$, S. Ottonello ${ }^{11}$, J. Palacios González ${ }^{5}$, G. Papalashviliti ${ }^{46}$, R. Papaleo ${ }^{20}$, C. Pastore ${ }^{36}$, A. M. Păun ${ }^{26}$, G.E. Păvălaşş, G. Pellegrinini ${ }^{17}$, C. Pellegrino ${ }^{18,58}$, M. Perrin-Terrin ${ }^{1}$, V. Pestel ${ }^{15}$, P. Piattelli ${ }^{20}$, C. Pieterse ${ }^{5}$, O. Pisanti ${ }^{6,7}$, C. Poirè ${ }^{14}$, V. Popa ${ }^{26}$, T. Pradier ${ }^{3}$, F. Pratolongo ${ }^{11}$, I. Probst ${ }^{13}$, G. Pühlhofer ${ }^{51}$, S. Pulvirenti' ${ }^{20}$, G. Quéméner ${ }^{43}$, N. Randazzo ${ }^{2}$, A. Rapicavoli ${ }^{34}$, S. Razzaque ${ }^{50}$, D. Real ${ }^{5}$, S. Reck ${ }^{13}$, G. Riccobene ${ }^{20}$, L. Rigalleau ${ }^{24}$, A. Romanov ${ }^{11,42}$, A. Rovelli ${ }^{20}$, J. Royon ${ }^{1}$, F. Salesa Greus ${ }^{5}$, D. F. E. Samtleben ${ }^{15,47}$, A. Sánchez Losa $^{36,5}$, M. Sanguineti ${ }^{11,42}$, A. Santangelo ${ }^{51}$, D. Santonocito ${ }^{20}$, P. Sapienza ${ }^{20}$, J. Schmelling ${ }^{15}$, J. Schnabel ${ }^{13}$, M. F. Schneider ${ }^{13}$, J. Schumann ${ }^{13}$, H. M. Schutte ${ }^{21}$, J. Seneca ${ }^{15}$, I. Sgura ${ }^{36}$, R. Shanidze ${ }^{46}$, A. Sharma ${ }^{52}$, A. Sinopoulou ${ }^{10}$, B. Spisso ${ }^{41,6}$, M. Spurio ${ }^{17,18}$, D. Stavropoulos ${ }^{10}$, J. Steijger ${ }^{15}$, S. M. Stellacci ${ }^{41,6}$, M. Taiuti ${ }^{11,42}$, F. Tatone ${ }^{36}$, Y. Tayalati ${ }^{16}$, E. Tenllado ${ }^{44}$, D. Tézier ${ }^{1}$, T. Thakore ${ }^{5}$, S. Theraube ${ }^{1}$, H. Thiersen $^{21}$, P. Timmer $^{15}$, S. Tingay ${ }^{37}$, S. Tsagkli $^{10}$, V. Tsourapis ${ }^{10}$, E. Tzamariudaki ${ }^{10}$, D. Tzanetatos ${ }^{10}$, C. Valieri ${ }^{17}$, V. Van Elewyck ${ }^{4,49}$, G. Vasileiadis ${ }^{53}$, F. Versari ${ }^{17,18}$, S. Viola ${ }^{20}$, D. Vivolo ${ }^{6,29}$, G. de Wasseige ${ }^{4}$, J. Wilms ${ }^{54}$, R. Wojaczyński ${ }^{48}$, E. de Wolf ${ }^{15,27}$, T. Yousfi $^{23}$, S. Zavatarelli ${ }^{11}$, A. Zegarelli ${ }^{30,8}$, D. Zito ${ }^{20}$, J. D. Zornoza ${ }^{5}$, J. Zúñiga ${ }^{5}$, N. Zywucka ${ }^{21}$.

${ }^{1}$ Aix Marseille Univ, CNRS/IN2P3, CPPM, Marseille, France.

${ }^{2}$ INFN, Sezione di Catania, Via Santa Sofia 64, Catania, 95123 Italy.

${ }^{3}$ Université de Strasbourg, CNRS, IPHC UMR 7178, F-67000 Strasbourg, France.

${ }^{4}$ Université de Paris, CNRS, Astroparticule et Cosmologie, F-75013 Paris, France.

${ }^{5}$ IFIC - Instituto de Física Corpuscular (CSIC - Universitat de València), c/Catedrático José Beltrán, 2, 46980 Paterna, Valencia, Spain. ${ }^{6}$ INFN, Sezione di Napoli, Complesso Universitario di Monte S. Angelo, Via Cintia ed. G, Napoli, 80126 Italy.

${ }^{7}$ Università di Napoli "Federico II", Dip. Scienze Fisiche "E. Pancini”, Complesso Universitario di Monte S. Angelo, Via Cintia ed. G, Napoli, 80126 Italy.

${ }^{8}$ INFN, Sezione di Roma, Piazzale Aldo Moro 2, Roma, 00185 Italy.

${ }^{9}$ Universitat Politècnica de Catalunya, Laboratori d'Aplicacions Bioacústiques, Centre Tecnològic de Vilanova i la Geltrú, Avda. Rambla Exposició, s/n, Vilanova i la Geltrú, 08800 Spain.

${ }^{10}$ NCSR Demokritos, Institute of Nuclear and Particle Physics, Ag. Paraskevi Attikis, Athens, 15310 Greece.

${ }^{11}$ INFN, Sezione di Genova, Via Dodecaneso 33, Genova, 16146 Italy.

${ }^{12}$ University of Granada, Dept. of Computer Architecture and Technology/CITIC, 18071 Granada, Spain.

${ }^{13}$ Friedrich-Alexander-Universität Erlangen-Nürnberg, Erlangen Centre for Astroparticle Physics, Erwin-Rommel-Straße 1, 91058 Erlangen, Germany.

${ }^{14}$ Universitat Politècnica de València, Instituto de Investigación para la Gestión Integrada de las Zonas Costeras, C/ Paranimf, 1, Gandia, 46730 Spain.

${ }^{15}$ Nikhef, National Institute for Subatomic Physics, PO Box 41882, Amsterdam, 1009 DB Netherlands.

${ }^{16}$ University Mohammed V in Rabat, Faculty of Sciences, 4 av. Ibn Battouta, B.P. 1014, R.P. 10000 Rabat, Morocco.

${ }^{17}$ INFN, Sezione di Bologna, v.le C. Berti-Pichat, 6/2, Bologna, 40127 Italy.

\footnotetext{
1also at Dipartimento di Fisica, INFN Sezione di Padova and Università di Padova, I-35131, Padova, Italy
} 
${ }^{18}$ Università di Bologna, Dipartimento di Fisica e Astronomia, v.le C. Berti-Pichat, 6/2, Bologna, 40127 Italy.

${ }^{19}$ KVI-CART University of Groningen, Groningen, the Netherlands.

${ }^{20}$ INFN, Laboratori Nazionali del Sud, Via S. Sofia 62, Catania, 95123 Italy.

${ }^{21}$ North-West University, Centre for Space Research, Private Bag X6001, Potchefstroom, 2520 South Africa.

${ }^{22}$ Instituto Español de Oceanografía, Unidad Mixta IEO-UPV, C/ Paranimf, 1, Gandia, 46730 Spain.

${ }^{23}$ University Mohammed I, Faculty of Sciences, BV Mohammed VI, B.P. 717, R.P. 60000 Oujda, Morocco.

${ }^{24}$ Subatech, IMT Atlantique, IN2P3-CNRS, Université de Nantes, 4 rue Alfred Kastler - La Chantrerie, Nantes, BP 2072244307 France.

${ }^{25}$ Università di Salerno e INFN Gruppo Collegato di Salerno, Dipartimento di Matematica, Via Giovanni Paolo II 132, Fisciano, 84084 Italy.

${ }^{26}$ ISS, Atomistilor 409, Măgurele, RO-077125 Romania.

${ }^{27}$ University of Amsterdam, Institute of Physics/IHEF, PO Box 94216, Amsterdam, 1090 GE Netherlands.

${ }^{28}$ TNO, Technical Sciences, PO Box 155, Delft, 2600 AD Netherlands.

${ }^{29}$ Università degli Studi della Campania "Luigi Vanvitelli", Dipartimento di Matematica e Fisica, viale Lincoln 5, Caserta, 81100 Italy.

${ }^{30}$ Università La Sapienza, Dipartimento di Fisica, Piazzale Aldo Moro 2, Roma, 00185 Italy.

${ }^{31}$ Università di Bologna, Dipartimento di Ingegneria dell'Energia Elettrica e dell'Informazione "Guglielmo Marconi", Via dell’Università 50, Cesena, 47521 Italia.

${ }^{32}$ Cadi Ayyad University, Physics Department, Faculty of Science Semlalia, Av. My Abdellah, P.O.B. 2390, Marrakech, 40000 Morocco.

${ }^{33}$ University of the Witwatersrand, School of Physics, Private Bag 3, Johannesburg, Wits 2050 South Africa.

${ }^{34}$ Università di Catania, Dipartimento di Fisica e Astronomia "Ettore Majorana", Via Santa Sofia 64, Catania, 95123 Italy.

${ }^{35}$ INFN, LNF, Via Enrico Fermi, 40, Frascati, 00044 Italy.

${ }^{36}$ INFN, Sezione di Bari, via Orabona, 4, Bari, 70125 Italy.

${ }^{37}$ International Centre for Radio Astronomy Research, Curtin University, Bentley, WA 6102, Australia.

${ }^{38}$ University of Bari, Via Amendola 173, Bari, 70126 Italy.

${ }^{39}$ University Würzburg, Emil-Fischer-Straße 31, Würzburg, 97074 Germany.

${ }^{40}$ Western Sydney University, School of Computing, Engineering and Mathematics, Locked Bag 1797, Penrith, NSW 2751 Australia.

${ }^{41}$ Università di Salerno e INFN Gruppo Collegato di Salerno, Dipartimento di Fisica, Via Giovanni Paolo II 132, Fisciano, 84084 Italy.

${ }^{42}$ Università di Genova, Via Dodecaneso 33, Genova, 16146 Italy.

${ }^{43}$ Normandie Univ, ENSICAEN, UNICAEN, CNRS/IN2P3, LPC Caen, LPCCAEN, 6 boulevard Maréchal Juin, Caen, 14050 France.

${ }^{44}$ University of Granada, Dpto. de Física Teórica y del Cosmos \& C.A.F.P.E., 18071 Granada, Spain.

${ }^{45}$ NIOZ (Royal Netherlands Institute for Sea Research), PO Box 59, Den Burg, Texel, 1790 AB, the Netherlands.

${ }^{46}$ Tbilisi State University, Department of Physics, 3, Chavchavadze Ave., Tbilisi, 0179 Georgia.

${ }^{47}$ Leiden University, Leiden Institute of Physics, PO Box 9504, Leiden, 2300 RA Netherlands.

${ }^{48}$ National Centre for Nuclear Research, 02-093 Warsaw, Poland.

${ }^{49}$ Institut Universitaire de France, 1 rue Descartes, Paris, 75005 France.

${ }^{50}$ University of Johannesburg, Department Physics, PO Box 524, Auckland Park, 2006 South Africa.

${ }^{51}$ Eberhard Karls Universität Tübingen, Institut für Astronomie und Astrophysik, Sand 1, Tübingen, 72076 Germany.

${ }^{52}$ Università di Pisa, Dipartimento di Fisica, Largo Bruno Pontecorvo 3, Pisa, 56127 Italy.

${ }^{53}$ Laboratoire Univers et Particules de Montpellier, Place Eugène Bataillon - CC 72, Montpellier Cédex 05, 34095 France.

${ }^{54}$ Friedrich-Alexander-Universität Erlangen-Nürnberg, Remeis Sternwarte, Sternwartstraße 7, 96049 Bamberg, Germany.

${ }^{55}$ Université de Haute Alsace, 68100 Mulhouse Cedex, France.

${ }^{56}$ University of Münster, Institut für Kernphysik, Wilhelm-Klemm-Str. 9, Münster, 48149 Germany.

${ }^{57}$ Utrecht University, Department of Physics and Astronomy, PO Box 80000, Utrecht, 3508 TA Netherlands.

${ }^{58}$ INFN, CNAF, v.le C. Berti-Pichat, 6/2, Bologna, 40127 Italy. 